\title{
PESQUISA OPERACIONAL: MAXIMIZAÇÃO DO LUCRO NA CRIAÇÃO DE UM ESTOQUE DE SEGURANÇA EM UMA EMPRESA DE MOTO ACESSÓRIOS
}

\author{
Delânio da Silva Oliveira (Universidade Federal do Ceará) delaniosilva@gmail.com \\ Iany de Assis Maia (Universidade Federal do Ceará) ianymaia35@ gmail.com \\ Lara Fernanda Cavalcante Nery (Universidade Federal do Ceará) larafnery@ gmailcom \\ João Felipe Sales de Almeida (Universidade Federal do Ceará) joaofsalesa@ gmail.com \\ Mágda Ferreira Maia Torre (Universidade Federal do Ceará) magdamaia23@gmail.com
}

\section{Resumo}

O desenvolvimento e a competitividade do mercado exigiram diversas modificações das empresas em relação aos critérios de desempenho e, principalmente, ganhadores de pedido. Com isso, buscando suprir as variações das demandas do mercado e aumentar a confiabilidade dos clientes em uma empresa fornecedora de moto peças, localizada em Russas-CE, percebeuse a necessidade da construção de um estoque de segurança. Para o auxílio e validação dos resultados, foi realizado um estudo interdisciplinar utilizando estratégias de operações e pesquisa operacional com intuito de obter soluções ótimas para o problema. Buscando esse fim, utilizou-se de modelos de programação linear que, por sua vez, foram identificados requisitos e restrições da situação para construção da equação, em seguida, aplicou-se no software Excel, usando a ferramenta Solver para a obtenção da simulação, a fim de auxiliar na tomada de decisão pelos gestores. Os objetivos do estudo foram alcançados, assim como a importância e facilidade de interdisciplinaridade da área de pesquisa operacional foi conhecida.

Palavras-Chaves: Mix de produtos. Programação linear.

\section{Introdução}

Devido à velocidade de desenvolvimento do mercado, e consequentemente a crescente concorrência entre as empresas, percebe-se que os critérios ganhadores de pedidos estão mudando, parâmetros que antes eram diferenciais, passaram a ser básicos na concepção dos consumidores. Com isso, a rapidez com que as empresas conseguem oferecer seus produtos aos clientes, tornou-se essencial.

Nesse sentido, as empresas buscam aprimorar a gestão de suas atividades para que consigam ser superiores aos seus concorrentes, traçando estratégias de operação que fidelizem os clientes à empresa, como a confiabilidade. Dessa forma, "O objetivo da estratégia de operações é 
garantir que os processos de produção e entrega de valor ao cliente sejam alinhados com a intenção estratégica da empresa" (CORRÊA; CORRÊA, 2012, p. 36)

Algumas ações como eliminação de desperdícios, aumento da automação dos processos ou até mesmo, a criação de um estoque de segurança, buscam atender às variações de demanda e as condições exigidas pelos consumidores. Tendo em vista todas essas práticas, é necessário a realização de um trade-off pelos tomadores de decisão da empresa para identificar as melhores medidas a serem tomadas.

Com isso, a pesquisa operacional através da aplicação de modelos matemáticos ajuda os gestores a diminuir as incertezas e os riscos dos processos, podendo ser usados problemas de programação linear, com o auxílio de softwares, como o Excel, e ferramentas como o Solver, pois, segundo Bazarra, Jarvis e Sherali (2010), a programação linear busca a otimização, maximização ou minimização, de uma função sujeita a restrições, que limitam a solução.

Por fim, o objetivo do estudo, em suma, é encontrar o melhor mix de produtos para a criação de um estoque de segurança no setor de capas para assentos em uma empresa de motopeças, com a utilização de condições pré-determinadas, como valor de investimento e capacidade produtiva.

\section{Referencial teórico}

\subsection{Pesquisa operacional}

Segundo Hillier e Lieberman (2013) a pesquisa operacional (PO) trata-se precisamente das pesquisas sobre operações, sendo aplicada a problemas para compreender as atividades e direcionar as decisões de uma organização, podendo ser utilizada em diversas áreas do controle e gestão, como logística, manufatura, financeiro.

Portanto, "A pesquisa operacional consiste na utilização de métodos de apoio na tomada de decisão" (BELFIORE; FÁVERO, 2013, p.2), com isso, de acordo com os mesmos autores, a definição dos objetivos está diretamente relacionada à tomada de decisão, com o intuito de minimizar incertezas e riscos relacionados ao processo, valorizando a eficácia e a qualidade das informações. Nesse sentido, existem modelos matemáticos que auxiliam na simulação de soluções para esses problemas.

\subsection{Programação linear}

Modelagem matemática, de acordo Barbosa (2009), busca a relação entre a realidade e a matemática, de forma que a segunda desvenda ou explica as leis da primeira, que auxilia na 
solução de problemas reais de forma precisa e geralmente rápida. Segundo Colin (2015) os problemas de programação linear garantem a mesma estrutura para os algoritmos e as entradas. Com isso, para o algoritmo de maximização pode-se utilizar a forma padrão, na figura 1 .

Figura 1 - Forma padrão do modelo de programação linear de maximização

$$
\text { Maximizar } Z=c_{1} x_{1}+c_{2} x_{2}+\ldots+c_{n} x_{n}
$$

Sujeito a :

$$
\begin{aligned}
& a_{11} x_{1}+a_{12} x_{2}+\ldots+a_{1 n} x_{n} \leq b_{1} \\
& a_{21} x_{1}+a_{22} x_{2}+\ldots+a_{2 n} x_{n} \leq b_{2} \\
& a_{m 1} x_{1}+a_{m 2} x_{2}+\ldots+a_{m n} x_{n} \leq b_{m} \\
& x_{1}, x_{2}, x_{3}, \ldots x_{n} \geq 0
\end{aligned}
$$

Fonte: LACHTERMACHER (2007, p. 19)

E para formulação de qualquer problema de programação linear precisa-se entender conceitos básicos, que qualquer modelo possui, apresentado no quadro 1 .

Quadro 1 - Definições Básicas De Um Problema De Programação Linear

\begin{tabular}{|c|l}
\hline $\begin{array}{c}\text { Característica } \\
\text { deciáveis de }\end{array}$ & $\begin{array}{l}\text { Definição } \\
\text { Paro as variáveis a serem determinadas pelo modelo, sendo os } \\
\text { valores viáveis, que atendem a função objetivo e as restrições. }\end{array}$ \\
\hline Função objetivo & $\begin{array}{l}\text { São os coeficientes que são constantes no modelo, ou seja, } \\
\text { não se alteram, são fixas para solução do problema. }\end{array}$ \\
\hline Restriçães & $\begin{array}{l}\text { Objetivo do modelo, pode ser de maximizar ou minimizar a formada por coeficientes conhecidos e variáveis de } \\
\text { decisão. }\end{array}$ \\
\hline $\begin{array}{l}\text { Rão as condições que limitam o modelo, podendo ser } \\
\text { restrições de igualdades e/ou desigualdades. As equações ou } \\
\text { acompanham as variáveis de decisão (do lado esquerdo), e } \\
\text { coeficientes de requisitos mínimos (do lado direito) a serem } \\
\text { atendidos. }\end{array}$ \\
\hline não-negatividade
\end{tabular} \begin{tabular}{l}
$\begin{array}{l}\text { Restrição para que o conjunto de valores viáveis sejam } \\
\text { positivos, ou seja, todos os valores das variáveis de decisão } \\
\text { sejam maiores que zero. }\end{array}$ \\
\hline
\end{tabular}

Fonte: Adaptado de BAZARRA; JARVIS; SHERALI (2010).

Alguns requisitos devem ser considerados para que a utilização da forma padrão seja atendida, manipulando a resolução do problema por programação linear. Portanto, segundo Belfiore e 
Fávaro (2013) esses requisitos estão relacionados aos termos independentes das restrições ser não negativos, todas as restrições devem estar representadas por equações lineares e apresentarem formas de igualdade e, por fim, as variáveis de decisão também devem ser não negativas.

Logo, a programação linear reproduz diversas situações, possuindo modelos conhecidos e préestabelecidos, como o de mix de produtos, que serve para encontrar quais as melhores quantidades de determinados itens devem ser produzidos por uma empresa, com objetivos específicos.

\subsection{Problema do mix de produtos}

Segundo Belfiore e Fávero (2013) os problemas de mix de produtos busca encontrar a quantidade ideal de produtos a serem fabricados, sem que seja necessário eliminar algum produto ou super produzir outro. Seus objetivos podem variar de acordo com a necessidade da empresa podendo ser para maximizar ou minimizar os resultados. Quando a quantidade de produtos determinada para a fabricação deve ser exclusivamente inteira, o problema é classificado como programação inteira (PI), que tem como alternativa de solução do problema relaxar ou eliminar as restrições de integridade das variáveis de decisão, que, por fim, recaem em uma programação linear.

\section{Metodologia}

\subsection{Caracterização da pesquisa}

O trabalho realizado na empresa de moto peças em Russas - CE, de acordo com o procedimento é classificado como estudo de caso, pois através de um estudo profundo de alguns objetos, possibilita um amplo conhecimento mediante as suas características (GIL, 2002). Quanto à sua natureza a pesquisa é aplicada, devido às práticas, de modo que os resultados são utilizados para a solução de problemas (MARCONI; LAKATOS, 2002). E a fim de auxiliar no desenvolvimento do trabalho, foram feitas pesquisas sobre pesquisa operacional e programação linear, em materiais como livros, artigos, revistas e monografias.

A pesquisa tem uma abordagem metodológica do tipo quantitativa, onde para obter conclusões de determinado fenômeno, os resultados baseiam-se em análise de dados que são quantificáveis (GERHARDT; SILVEIRA, 2009). E para alcance do objetivo, de acordo com Gil (2008), a pesquisa é de caráter exploratório, que possibilita a influência de várias informações sobre o assunto, para uma maior confiança acerca do problema, com a finalidade de desenvolver ideias a partir da criação de hipóteses. 
A caracterização da pesquisa, pode ser visualizada em síntese na figura abaixo.

Figura 2 - Caracterização da pesquisa

\begin{tabular}{|c|l|}
\hline $\begin{array}{c}\text { Quanto ao } \\
\text { procedimento }\end{array}$ & $\begin{array}{l}\text { • Estudo de caso } \\
\text { • Pesquisas bibliográficas }\end{array}$ \\
\hline $\begin{array}{c}\text { Quanto à } \\
\text { natureza }\end{array}$ & • Aplicada \\
\hline $\begin{array}{c}\text { Quanto à } \\
\text { abordagem }\end{array}$ & • Quantitativa \\
\hline $\begin{array}{c}\text { Quanto ao } \\
\text { caráter }\end{array}$ & • Exploratório \\
\hline
\end{tabular}

Fonte: Autores (2020)

\subsection{Métodos e procedimentos}

Inicialmente foram realizadas visitas a empresa com o intuito de observar o dia a dia e identificar os problemas para desenvolver um estudo de melhorias. Com isso, alguns problemas foram levantados junto aos responsáveis, como a disposição dos equipamentos, os tempos de processos ou até mesmo os tempos em que os produtos eram finalizados e entregues ao cliente.

Em seguida, para auxiliar no desenvolvimento do estudo foram realizadas pesquisas sobre programação linear e modelos que melhor se adaptam a situação. Por fim, o modelo escolhido foi aplicado a ferramenta Solver, no software Excel.

\subsection{Caracterização da empresa}

O estudo foi realizado em uma empresa que fabrica peças e acessórios para motos e bicicletas, usados para manutenção. Atua no mercado desde 2005, e hoje se localiza no bairro Lagoa do Toco, em Russas, Ceará, com a distribuição em todo território nacional. Trata-se de uma empresa privada, com 110 colaboradores trabalhando diretamente ligados ao chão de fábrica, 8 em funções administrativas e 12 representantes comerciais.

A empresa é dividida em dois processos produtivos, que são a produção de peças metálicas e a produção de capas para coberta de bancos, e a programação é puxada, onde a fabricação acontece conforme há uma requisição do mercado (NAZARENO, 2008). Sendo assim, o processo de fabricação começa com o encaminhamento de ordens de serviço, as quais partem da administração de acordo com o que é repassado pela equipe de vendas. 
O processo de produção dessas capas inicia-se com o corte do couro sintético, o qual é disposto em uma mesa que possui um gabarito com marcações. Este processo é realizado manualmente, utilizando uma navalha acoplada a um cano de metal, que facilita o manuseio pelo operador, a cada repetição dessa tarefa são cortadas 15 unidades do produto. Nas etapas seguintes é processado um produto de cada vez, posteriormente ocorre a estampagem em relevo, a qual é realizada por uma máquina de frequência, seguindo as etapas de vulcanização e resfriamento.

Simultaneamente, são confeccionados os adesivos de plastisol, que também são feitos manualmente, passando por três etapas, o preenchimento dos moldes com a matéria-prima, o cozimento e o resfriamento dos adesivos. Após os adesivos serem produzidos é realizado um corte e retirada dos excessos de material, adequando-os para serem fixados nas capas. Na última etapa os adesivos são fixos nas capas por uma prensa, em seguida, resfriam e são levados para embalagem e expedição.

O processo descrito pode ser visualizado de forma mais detalhada no mapeamento da figura 3 abaixo.

Figura 3 - Mapeamento do processo produtivo de capas para assentos de motos

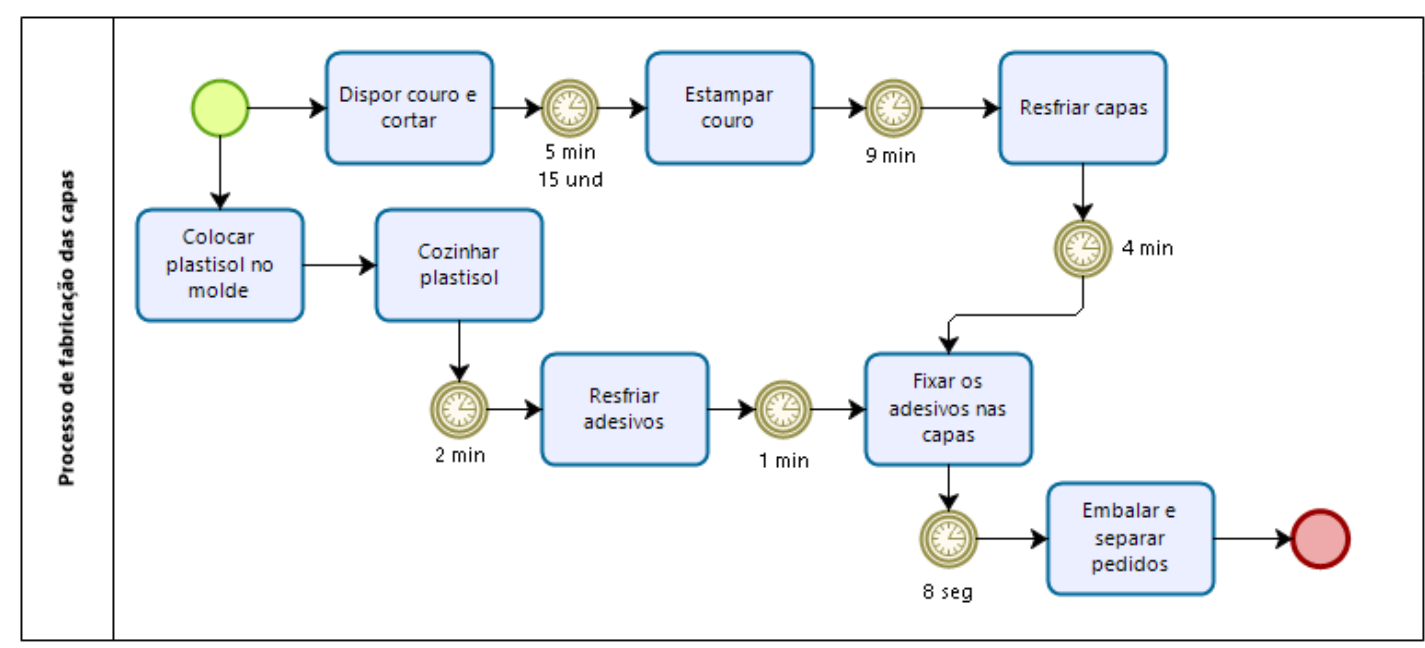

Fonte: Autores (2020)

\section{Resultados e discussões}

Com a análise da situação da empresa, foi percebida uma deficiência no processo produtivo de capas para assentos de motos, que devido à falta de estoque para esse setor produtivo, prejudicava na rapidez da separação e entrega dos pedidos dos clientes, em razão da espera para a produção das quantidades requeridas.

Assim, para iniciar o desenvolvimento do estudo, utilizou-se a forma padrão para maximização do lucro, na qual foi aplicada a um problema de mix de produtos, com o intuito encontrar a 
quantidade ideal de capas de cada modelo. Com isso, para que o modelo do problema seja solucionado, as condições do quadro seguinte são consideradas.

Quadro 2 - Condições do problema

\begin{tabular}{|c|c|}
\hline $\begin{array}{c}\text { Cunção Objetivo } \\
\text { (F.O.) }\end{array}$ & Tipos de capas produzidas. [P] \\
\hline \multirow{7}{*}{$\begin{array}{c}\text { Variáveis de } \\
\text { Decisão }\end{array}$} & Máx $(z)=\sum_{p \in P} X p, L p, \forall p \in P$ \\
onde, $L p$ é o lucro obtido com o produto $p$
\end{tabular}

Fonte: Autores (2020)

Em seguida foram coletados os valores do custo de fabricação de cada modelo de capa e seus respectivos preços de venda, sendo essas as variáveis que auxiliarão a decisão. No processo produtivo são encontradas algumas restrições, como as capacidades da produção, e o total de investimento destinado à construção desse estoque, o que pode ser melhor observado no quadro 3. 


\begin{tabular}{|c|c|}
\hline \multicolumn{2}{|c|}{ Restrições } \\
\hline Investimento Máx. & $\mathrm{R} \$ \quad 100.000,00$ \\
\hline Produção Min. & 500 \\
\hline Produção Máx. & 4000 \\
\hline Prod. Total Máx. & 20000 \\
\hline
\end{tabular}

Fonte: Autores (2020)

De acordo com as condições estabelecidas, os dados foram organizados em planilhas no Excel, e aplicados ao Solver, suplemento do software que utiliza o algoritmo Simplex para encontrar a solução ótima de um problema de programação linear. Como pode ser visto no quadro 4.

Quadro 4 - Dados do problema

\begin{tabular}{|c|lr|lr|c}
\hline Tipos de capas & \multicolumn{2}{|l|}{ Custo Unitário } & \multicolumn{2}{l|}{ Preço de Venda } & Lucro \\
\hline 3D Pintura Degradê & $\mathrm{R} \$$ & 13,00 & $\mathrm{R} \$$ & 15,00 & $\mathrm{R} \$ 2,00$ \\
\hline 3D Drawing & $\mathrm{R} \$$ & 12,50 & $\mathrm{R} \$$ & 15,00 & $\mathrm{R} \$ 2,50$ \\
\hline Antiderrapante & $\mathrm{R} \$$ & 10,00 & $\mathrm{R} \$$ & 12,00 & $\mathrm{R} \$ 2,00$ \\
\hline Multi Lateral & $\mathrm{R} \$$ & 12,00 & $\mathrm{R} \$$ & 15,00 & $\mathrm{R} \$ 3,00$ \\
\hline Multi Color & $\mathrm{R} \$$ & 11,00 & $\mathrm{R} \$$ & 12,00 & $\mathrm{R} \$ 1,00$ \\
\hline
\end{tabular}

Fonte: Autores (2020)

Com isso, foi possível encontrar o mix ideal de produtos, mostrado no quadro 5 abaixo, ou seja, a quantidade de capas de cada tipo para que fosse obtido o maior lucro possível na criação do novo estoque.

Quadro 5 - Quantidade ideal de cada tipo de capas

\begin{tabular}{|c|rr|r}
\hline Tipos de capas & \multicolumn{2}{|c|}{ Lucro } & Quantidades \\
\hline 3D Pintura Degradê & $\mathrm{R} \$$ & 2,00 & 500 \\
\hline 3D Drawing & $\mathrm{R} \$$ & 2,50 & 2800 \\
\hline Antiderrapante & $\mathrm{R} \$$ & 2,00 & 500 \\
\hline Multi Lateral & $\mathrm{R} \$$ & 3,00 & 4000 \\
\hline Multi Color & $\mathrm{R} \$$ & 1,00 & 500 \\
\hline \multicolumn{2}{|c|}{ Total } & 8300
\end{tabular}

Fonte: Autores (2020)

Dadas as quantidades de cada tipo a serem produzidas seria possível, como mostrado no quadro 5 , chegar a um lucro total de quase $18 \%$ do faturado ao vender todos os produtos, considerado um valor razoável, tendo em vista todas as variáveis do processo. Os valores são mostrados no quadro 6.

Quadro 6 - Faturamento e lucro estimados

\begin{tabular}{|c|rr|}
\hline Lucro Total (F.O.) & $\mathrm{R} \$$ & $21.500,00$ \\
\hline Custo Total & $\mathrm{R} \$$ & $100.000,00$ \\
\hline Faturamento & $\mathrm{R} \$$ & $121.500,00$
\end{tabular}




\section{Considerações finais}

Referindo-se inicialmente ao que diz respeito à pesquisa operacional consideram-se os resultados satisfatórios, tendo em vista que o objetivo de estipular, de forma mais coerente e real, os valores que encontram a maximização dos lucros, para a criação de um estoque de segurança, foi atendido.

Percebe-se também que essa área de estudo possui uma interdisciplinaridade com diversas vertentes da engenharia de produção, como a de gestão de custo, que foi um dos focos deste estudo. A importância da utilização da PO como um auxílio na tomada de decisão também possui uma relevância, vistas as diversas formas de simulação que facilitam nas escolhas dos gestores de uma organização.

Por fim, propõe como trabalhos futuros a junção dessa ciência com outras áreas de gestão e desenvolvimento, com objetivos variados, sejam de maximização ou minimização de riscos, incertezas e complexidades do processo, assim como pôr em prática o que foi feito com as demais linhas de produtos oferecidos pela empresa.

\section{Referências bibliográficas}

BARBOSA, Jonei Cerqueira. Modelagem e modelos matemáticos na educação científica. ALEXANDRIA: Revista de Educação Em Ciência e Tecnologia, v.2, n.2, p. 69-85. jul, 2009.

BAZARAA, Mokhtar S.; JARVIS, John J.; SHERALI, Hanif D. Linear programming and network flows. 4 ed. Nova Jersey, EUA, 2011, 748 p.

COLIN, Emerson Carlos. Pesquisa operacional: 170 aplicações em estratégia, finanças, logística, produção, merketing e vendas . Rio de Janeiro: LTC, c2007. xix, 501 p. ISBN 9788521615590 (broch.).

CORRÊA, Henrique L.; CORRÊA, Carlos A. Administração de produção e operações: uma abordagem estratégica. 3. ed. São Paulo: Atlas, 2012.

FÁVERO, Luiz Paulo; BELFIORE, Patrícia. Pesquisa operacional para os cursos de engenharia. Rio de Janeiro: Elsevier, 2013. 541 p.

GERHARDT, Tatiana Engel; SILVEIRA, Denise Tolfo Silveira. Métodos de pesquisa. Universidade Aberta do Brasil - UAB/UFRGS. Porto Alegre: Editora da UFRGS, 2009. 120 p.

GIL, Antônio Carlos. Como elaborar projetos de pesquisa. 4. ed. São Paulo: Editora Atlas, 2002. 171 p. 
GIL, Antônio Carlos. Métodos e técnicas de pesquisa social. 6. ed. São Paulo: Editora Atlas, 2008. 200 p.

HILLIER, Frederick S.; LEIBERMAN, Gerald J. Introdução à pesquisa operacional. 9 ed. Porto Alegre: AMGH Editora, 2013. 1005 p.

LACHTERMACHE, Gerson. Pesquisa operacional na tomada de decisões: modelagem em Excel. 3. ed. Rio de Janeiro: Elsevier. 2007. 213 p.

MARCONI, Marina de Andrade; LAKATOS, Eva Maria. Técnicas de pesquisa: planejamento e execução de pesquisas, amostragens e técnicas de pesquisas, elaboração, análise e interpretação de dados. 5. ed. São Paulo: Editora Atlas, 2002. 282 p.

NAZARENO, Renato Renovato. Desenvolvimento de sistemas híbridos de planejamento e programação da produção com foco na implantação da manufatura enxuta. Tese (Doutorado em Engenharia de Produção). Escola de Engenharia de São Carlos - Universidade de São Paulo (USP). São Carlos/SP, 2008. 314 p. 